\title{
Gravity-Induced Flocculation
}

\author{
D. H. MELIK ${ }^{1}$ AND H. S. FOGLER \\ Department of Chemical Engineering, The University of Michigan, Ann Arbor, Michigan 48109
}

Received October 7, 1983; accepted February 14, 1984

\begin{abstract}
In polydisperse colloidal systems, floceulation can occur as a result of the differential creaming rates between small and large particles. As an improvement on earlier work, we have rigorously modelled this process of gravity-induced flocculation by incorporating gravitational and interparticle (both attractive and repulsive) forces, as well as hydrodynamic interactions in our analysis. From this analysis capture cross-sections and collision frequencies can be precisely defined and computed. In the absence of electrostatic repulsion, the gravity-induced flocculation rate is approximately proportional to $g^{0.80}$ (where $g$ is the local acceleration of gravity) and not $g$ as previously predicted. When electrostatic repulsion is significant, particles can flocculate into either a primary or secondary minimum (as described by DLVO theory), or remain dispersed. The possibility of two different types of doublets leads to an interesting phenomenon. When Brownian motion can be neglected, dilute spherical sols can be unstable at low and high gravitational forces, but stable against gravity-induced flocculation at intermediate values.
\end{abstract}

\section{INTRODUCTION}

While there has been an extensive and rigorous treatment of both Brownian and shearinduced flocculation (cf. 1-10), there has been no analogous study of particle/particle aggregation due to sedimentation (gravity-induced) flocculation in polydisperse colloidal systems. Gravity-induced flocculation arises from the differential creaming rates between large and small particles. The faster creaming large particles eventually sweep out the slower creaming small particles in their path, leading to possible flocculation if interparticle interactions are favorable.

Saffman and Turner (11) were the first to consider the case of gravity-induced flocculation. In their analysis, interparticle forces were ignored, and particles were assumed to follow rectilinear trajectories until contact and to stick irreversibly upon contact. The resulting single-particle capture rate was found to be

$$
\not_{\mathrm{Gr}}^{0}=\pi\left(u_{02}-u_{01}\right)\left(a_{1}+a_{2}\right)^{2} N_{02},
$$

\footnotetext{
${ }^{1}$ Present address: the Procter \& Gamble Company, Miami Valley Laboratories, Cincinnati, Ohio 45247.
}

where $u_{0 i}$ is Stokes creaming velocity for particles of radius $a_{i}$, and $N_{02}$ the bulk particle concentration of particles of radius $a_{2}$. Since all resistances are ignored in this analysis, Saffman and Turner's flocculation rate provides a useful scale upon which other flocculation rates can be compared. The ratio of Saffman and Turner's flocculation rate to the actual flocculation rate in a system undergoing gravity-induced flocculation, $\delta_{\mathrm{Gr}}$, is known as the stability ratio,

$$
W_{\mathrm{Gr}}=\frac{\beta_{\mathrm{Gr}}^{0}}{\partial_{\mathrm{Gr}}} .
$$

The gravity-induced capture efficiency is defined as the reciprocal of the stability ratio,

$$
\alpha_{\mathrm{Gr}}=\frac{1}{W_{\mathrm{Gr}}} .
$$

Extending Saffman and Turner's work by including hydrodynamic interactions leads to the curious prediction that particle capture is impossible (12). This result is a consequence of the hydrodynamic force becoming infinite as the particle separation becomes zero. This problem is usually circumvented by assuming that particle capture occurs once the particles 
have reached some prespecified separation (cf. 13-17). However, the sensitivity of this assumption (13) leads one to question the prudence of neglecting interparticle forces in the analysis.

Even when one considers the simultaneous effects of interparticle forces and hydrodynamic interactions, particle capture may not occur. The interplay between these two forces has been initially studied in conjunction with the problem of particle capture on spherical collectors $(18,19)$. However, in these studies the collector is always much larger than the particle so only sphere-plane interactions (surface and hydrodynamic) were considered. Consequently, particle size ratio effects were excluded. In addition, no stability criteria delineating favorable and unfavorable capture conditions were presented even though no particle capture was observed under certain electrostatic conditions.

The objective of this paper is to rigorously describe the simultaneous effects of gravitational, interparticle (in particular, London van der Waal attractive and electric double layer repulsive), and hydrodynamic forces on the process of gravity-induced flocculation. This will be accomplished in two ways:

(i) Stability criteria will be outlined and used to determine under which chemical and physical conditions either complete stability or instability from sedimentation flocculation is possible; and

(ii) The effects of important system parameters (e.g., acceleration of gravity, particle size ratio, electrolyte concentration, etc.) on the gravity-induced capture efficiency will be discussed.

Whenever possible comparisons will be made between our results and those of other investigators. In particular, the processes of gravity- and shear-induced flocculation will be compared. As will be shown, these two processes have qualitatively similar behavior under certain conditions, and widely different behavior under other conditions.

\section{THEORY}

A trajectory model for the gravity-induced flocculation of dilute suspensions of polydisperse colloidal spheres in quiescent media is described in this section. Because the suspension is assumed to be dilute, only two-particle interactions are included in the analysis.

Since colloidal sized particles are in the low Reynolds number regime, the governing hydrodynamic equations of motion are linear (20). Consequently, the relative velocity of the two spheres shown in Fig. 1 can be decomposed into separate contributions, namely (21)

$$
\mathbf{v}=\mathbf{v}_{\mathrm{g}}+\mathbf{v}_{\text {int }}+\mathbf{v}_{\mathrm{Br}},
$$

where $v_{\mathrm{g}}$ is the relative velocity due to gravitational forces, $\mathbf{v}_{\text {int }}$ the relative velocity due to interparticle forces, and $\mathbf{v}_{\mathrm{Br}}$ the relative velocity due to Brownian diffusion. For the analysis in this paper we are only concerned about the case of negligible Brownian diffusion. Therefore, $\mathbf{v}_{\mathrm{Br}}=0$ and the trajectory of the particle pair is uniquely determined from an initial configuration. Brownian diffusion has been included in a separate analysis (21).

The trajectories are described in a moving coordinate system $(r, \theta, \phi)$ with a fixed orientation in space and the origin embedded in the center of the smaller reference sphere (see Fig. 1). The trajectory equations are given by the spherical components of the relative velocity $\mathbf{v}=\mathbf{v}_{\mathrm{g}}+\mathbf{v}_{\text {int }}$. These velocity components are (21)

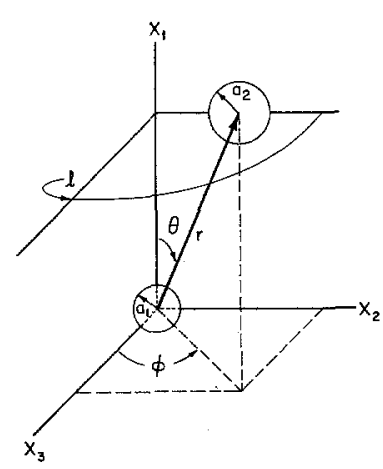

FIG. 1. Binary encounter between different sized spheres leading to particle capture. 


$$
\begin{gathered}
V_{\mathrm{r}}=\frac{d r}{d t}=-\left(u_{02}-u_{01}\right) \mathcal{A}(r) \cos \theta \\
-\frac{\mathcal{g}(r) D_{0}}{k T} \frac{\partial V_{\mathrm{int}}}{\partial r}, \\
V_{\theta}=r \frac{d \theta}{d t}=\left(u_{02}-u_{01}\right) \mathcal{B}(r) \sin \theta,
\end{gathered}
$$

and

$$
V_{\phi}=r \sin \theta \frac{d \phi}{d t}=0
$$

In [5] $\mathcal{A}(r)$ and $\mathscr{B}(r)$ are the hydrodynamic corrections to Stokes relative creaming rate $\left(u_{02}-u_{01}\right), \mathcal{S}(r)$ the hydrodynamic correction to Stokes-Einstein relative diffusion coefficient $D_{0}, V_{\text {int }}$ the total interparticle potential, $k$ the Boltzmann constant, $T$ the absolute temperature, and $t$ the time. It should be noted that the hydrodynamic corrections depend on both the distance between particle centers $r$ and the particle size ratio $\lambda=a_{1} / a_{2}$ (note: by definition $0<\lambda \leqslant 1$ ).

Far upstream from the reference sphere one can define a capture cross-section. All spheres whose centers pass through this capture crosssection will follow what is normally called a "closed" trajectory, and will ultimately be captured by the reference sphere. Spheres which do not pass through this cross-section follow an "open" trajectory, and will not be captured. A "limiting" or "critical" trajectory separates the regions of open and closed trajectories. By determining the separation $\ell$ between the critical trajectory and the centerline of the reference sphere as $r \rightarrow \infty$ (see Fig. 1), one can determine the capture cross-section. Due to the symmetry of gravitational motion about the $X_{1}$ axis the capture cross-section is always circular, so that the separation $\ell$ is the radius of this limiting area.

The single-particle gravity-induced flocculation (capture) rate can then be determined from the net flux of particles passing through the capture cross-section, namely

$$
\not_{\mathrm{Gr}}=\pi \ell^{2}\left(u_{02}-u_{01}\right) N_{02},
$$

where $\left(u_{02}-u_{01}\right)$ is the relative particle velocity for infinite separation since $v_{\text {int }} \rightarrow 0$ as $r \rightarrow$ $\infty$. From [1]-[3] and [6] it follows that the gravity-induced capture efficiency is given by

$$
\alpha_{\mathrm{Gr}}=\left\{\frac{\ell}{a_{1}+a_{2}}\right\}^{2} .
$$

In order to use the trajectory equations [5] to determine the capture cross-section defined by $\ell$, and therefore compute gravity-induced capture efficiencies, one must assume a form of the total interparticle potential $V_{\text {int }}$. The most commonly used model is described by DLVO theory $(22,23)$, which assumes that the total interparticle potential is obtained by summing the attractive and repulsive contributions, namely

$$
V_{\mathrm{int}}=V_{\mathrm{A}}+V_{\mathrm{R}} \text {. }
$$

The most important feature of the DLVO theory is the presence of either a primary minimum or both a secondary and primary minimum in the potential energy function.

The London van der Waal attraction between unequal spheres for unretarded potentials was given by Hamaker (24)

$$
\begin{aligned}
V_{\mathrm{A}}= & -\frac{2 A a_{1} a_{2}}{3\left(a_{1}+a_{2}\right)^{2}}\left\{\frac{2}{R^{2}-4}\right. \\
& +\frac{2}{R^{2}-4(1-\lambda)^{2} /(1+\lambda)^{2}}+\frac{(1+\lambda)^{2}}{4 \lambda} \\
& \left.\times \ln \left(\frac{R^{2}-4}{R^{2}-4(1-\lambda)^{2} /(1+\lambda)^{2}}\right)\right\},
\end{aligned}
$$

where $R=2 r /\left(a_{1}+a_{2}\right)$ is the dimensionless interparticle distance, and $A$ the Hamaker constant. For retarded attractive potentials, the results of Ho and Higuchi (25) are applicable, namely

$$
V_{\mathrm{A}} \simeq-\frac{A a_{1} a_{2}}{3\left(a_{1}+a_{2}\right)^{2}(R-2)} Z\left(p_{0}\right),
$$

where

$$
Z\left(p_{0}\right)=\frac{1}{1+1.7692 p_{0}} \text { for } p_{0} \leqslant 1.0
$$




$$
=\frac{2.45}{5 p_{0}}-\frac{2.17}{15 p_{0}^{2}}+\frac{0.59}{35 p_{0}^{3}} \text { for } p_{0}>1.0
$$

with $p_{0}=2 \pi(R-2) / \nu$, and $\nu=2 \lambda_{\mathrm{L}} /\left(a_{1}+a_{2}\right)$. The dimensionless parameter $\nu$ determines the degree of retardation where the characteristic London wavelength of the atoms, $\lambda_{\mathrm{L}}$, is typically $10^{-5} \mathrm{~cm}(26)$.

The electrostatic repulsion potential between unequally sized spheres is given by Hogg et al. (27)

$$
\begin{aligned}
V_{\mathrm{R}}= & \frac{\epsilon a_{1} a_{2} \Psi_{0}^{2}}{\left(a_{1}+a_{2}\right)} \\
& \times \ln \{1+\exp [-\kappa(R-2)]\},
\end{aligned}
$$

where $\kappa=K\left(a_{1}+a_{2}\right) / 2$ is the dimensionless reciprocal of the Debye-Hückel double layer thickness, $\epsilon$ the dielectric constant of the medium, and $\Psi_{0}$ the surface potential. Equation [12] is valid only for thin double layers $(\kappa$ $\gg 1$ ), low surface potentials $\left(\Psi_{0}<\simeq 25 \mathrm{mV}\right.$ ), symmetrical electrolytes, and constant surface potentials. It has recently (28) been argued that due to geometrical approximations used in deriving [12], [12] is valid only when $\lambda$ $\simeq 1$, with the error increasing exponentially as $\lambda$ approaches zero. However, Ohshima $e t$ al. (29) have more recently shown that there are no geometrical limitations in [12], so that [12] is exact to an order of $1 / \kappa$ for all $\lambda$.

Introducing the dimensionless force functions $f_{\mathrm{A}}(R, \nu)$ given by

$$
f_{\mathrm{A}}=\frac{2 k T\left(a_{1}+a_{2}\right)^{2}}{A a_{1} a_{2}} \frac{\partial V_{\mathrm{A}}}{\partial R},
$$

and $f_{\mathrm{R}}(R, \kappa)$ given by

$$
f_{\mathrm{R}}=\frac{k T\left(a_{1}+a_{2}\right)}{\epsilon \Psi_{0}^{2} a_{1} a_{2}} \frac{\partial V_{\mathrm{R}}}{\partial R},
$$

the total dimensionless interparticle force $F_{\text {int }}$ can be expressed as

$$
F_{\mathrm{int}}=-\frac{1}{k T} \frac{\partial V_{\mathrm{int}}}{\partial R}=-N_{\mathrm{A}}\left[f_{\mathrm{A}}-N_{\mathrm{R}} f_{\mathrm{R}}\right],
$$

where the dimensionless London van der Waal attractive number is given by

$$
N_{\mathrm{A}}=\frac{2 A a_{1} a_{2}}{k T\left(a_{1}+a_{2}\right)^{2}}=\frac{2 A}{k T} \frac{\lambda}{(1+\lambda)^{2}},
$$

and the dimensionless electrostatic repulsive number is given by

$$
N_{\mathrm{R}}=\frac{\epsilon \Psi_{0}^{2}\left(a_{1}+a_{2}\right)}{2 A} .
$$

The repulsion number describes the relative importance of repulsive to attractive interparticle forces. The behavior of the total interparticle force can be best illustrated by looking at the maximum net repulsive force. This maximum net repulsive force is defined as

$$
\left(\frac{F_{\mathrm{int}}}{N_{\mathrm{A}}}\right)_{\max }=\max \left\{N_{\mathrm{R}} f_{\mathrm{R}}-f_{\mathrm{A}}\right\},
$$

and can be determined by computing the smallest interparticle distance (for a given $\nu$, $\kappa$, and $N_{\mathrm{R}}$ ) at which

$$
\frac{1}{N_{\mathrm{A}}} \frac{\partial F_{\mathrm{int}}}{\partial R}=0 .
$$

Figure 2 shows the effect of increasing the electrolyte concentration $C\left(C \propto \kappa^{2}\right)$ on the maximum net repulsive force for various surface potentials $\Psi_{0}\left(\Psi_{0} \propto N_{\mathrm{R}}^{1 / 2}\right)$. It is evident from this figure that for low values of $k$, in-

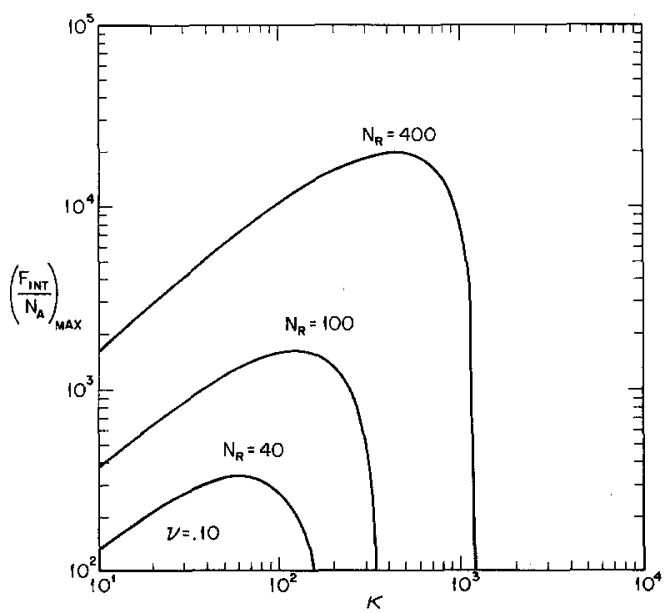

FIG. 2. Effect of increasing electrolyte concentration on the maximum net repulsive force for various surface potentials. 
creasing $\kappa$ results in an increase in the maximum repulsive force. Whereas, for large values of $\kappa$, increasing $\kappa$ results in a decrease in the maximum repulsive force. Consequently, flocculation may be possible at low and high values of $\kappa$, but impossible at intermediate values. This conclusion, of course, depends on the magnitude of the net gravitational force. However, as will be shown, the presence of this maximum leads to some interesting results.

Substituting [15] into the velocity equations [5], dividing the radial component by the tangential component to obtain the reduced trajectory equation $d R / d \theta$, and dedimensionalizing results in

$$
\frac{d R}{d \theta}=\frac{-R\left\{\mathcal{A}(R) \cos \theta+\frac{\mathcal{S}(R)}{N_{\mathrm{G}}}\left[f_{\mathrm{A}}-N_{\mathrm{R}} f_{\mathrm{R}}\right]\right\}}{\mathscr{B}(R) \sin \theta},
$$

where

$$
\begin{aligned}
N_{\mathrm{G}} & =\frac{\left(u_{02}-u_{01}\right)\left(a_{1}+a_{2}\right)}{2 N_{\mathrm{A}} D_{0}} \\
& =\frac{\pi g \Delta \rho a_{2}^{4}}{3 A}(1+\lambda)^{2}\left(1-\lambda^{2}\right),
\end{aligned}
$$

with $g$ being the local acceleration of gravity and $\Delta \rho$ the density difference between the particles and the suspending medium. The dimensionless parameter $N_{\mathrm{G}}$ describes the relative importance of gravitational to attractive forces.

\section{RESULTS AND DISCUSSION}

\section{Computation of the Capture Cross-Section}

The reduced trajectory equation [20] was numerically integrated using Gear's predictorcorrector method with an upstream initial condition of $R_{0}=20$. For separations $R$ $\geqslant 20$, particle/particle interactions are negligible, and the trajectories follow the undisturbed streamlines. By systematically varying the initial condition on $\theta$, the limiting value of $\theta, \theta_{\text {lim }}$, for which particle capture occurs can be determined. Once $\theta_{\text {lim }}$ is found, the dimensionless radius of the capture cross-section $\ell^{*}\left[=2 \ell /\left(a_{1}+a_{2}\right)\right]$ is known, since $\ell^{*}$ $=R_{0} \times \sin ^{-1} \theta_{\text {lim }}$.

The calculations of a trajectory were stopped when either a permanent doublet (primary or secondary) was formed or when the larger sphere passed sufficiently far downstream after an encounter with the smaller sphere. In order to avoid numerical difficulties associated with the formation of primary doublets ( $V_{\text {int }} \rightarrow-\infty$ as $R \rightarrow 2$ ), it was assumed that once the particles reached an interparticle distance $R=2.0001$, a primary doublet was formed. This assumption is equivalent to including Born repulsion in the total interparticle force expression. Born repulsion limits the separation between particle surfaces to some minimum value.

The hydrodynamic corrections $\mathcal{A}(R)$ and $\mathscr{S}(R)$ can be easily computed for any interparticle distance (except $R=2$ ) and particle size ratio using the explicit series solution given by Spielman (2). On the other hand, the hydrodynamic correction $\mathcal{B}(R)$ is difficult to compute and only results for specific interparticle distances and particle size ratios can be derived from the numerical computations of Davis (30) and O'Neill and Majumdar (31). Consequently, a two-dimensional interpolating routine was devised to calculate all three hydrodynamic corrections for any particle size ratio between 0 and 1 and interparticle distance between 2.0 and 5.0. For $R>5.0$, farfield asymptotic expressions were used. (Note: for more details on calculating $\mathcal{A}(R), \mathscr{B}(R)$, and $S(R)$ see ref. 32 .)

\section{Stability Criteria}

In this section stability criteria are discussed which allow one to determine under which conditions gravity-induced flocculation is favorable.

In order for two different sized spheres to be completely stable against gravity-induced flocculation, the capture cross-section must vanish, i.e., $\alpha_{\mathrm{Gr}}=0$. This requires that particle 
capture does not even occur for a pair whose line-of-centers is aligned with gravity (i.e., along the $X_{1}$ axis) at $R=\infty$.

For this case the critical trajectory has the initial conditions $\theta=0$ and $R=\infty$. Since gravitational motion is symmetric about the $X_{1}$ axis, $d \theta / d t=0$ for this trajectory and the particles will approach one another until either secondary or primary minimum flocculation occurs. For stability against primary minimum flocculation, the net gravitational force $N_{\mathrm{G}}$ bringing the particles together must be less than the maximum net repulsive force keeping the particles separated. This condition is met if the radial velocity component vanishes at some separation greater than $R=2$. From [5a] and [15], we have

$\frac{d R}{d \tau}=-\mathcal{A}(R) \cos \theta-\frac{\mathcal{S}(R)}{N_{\mathrm{G}}}\left[f_{\mathrm{A}}-N_{\mathrm{R}} f_{\mathrm{R}}\right]$,

where the dimensionless time $\tau=2 t\left(u_{02}\right.$ $\left.-u_{01}\right) /\left(a_{1}+a_{2}\right)$. It follows from [22] that if

$$
N_{\mathrm{G}}<\max \left\{\frac{g(R)}{\mathcal{A}(R)}\left[N_{\mathrm{R}} f_{\mathrm{R}}-f_{\mathrm{A}}\right]\right\},
$$

then $d R / d \tau=0$ at some separation larger than $R=2$. Under these conditions primary minimum flocculation will not occur, although flocculation in the secondary minimum is possible.

Once a particle pair has flocculated, they can either remain flocculated or redisperse. To determine whether deflocculation is possible, the alignment of the particle pair with the principal straining axis $\theta=\pi$ is considered. Under these conditions, gravity is pulling the particles apart. Consequently, if the net gravitational force pulling the flocculated particles apart is greater than the maximum net attractive force keeping the pair flocculated, deflocculation will occur. This condition is only met if $d R / d \tau>0$. From [22] it follows that if

$$
N_{\mathrm{G}}>\max \left\{\frac{g(R)}{\mathcal{A}(R)}\left[f_{\mathrm{A}}-N_{\mathrm{R}} t_{\mathrm{R}}\right]\right\},
$$

then $d R / d \tau>0$ and deflocculation will occur. The term in brackets may have two roots, corresponding to the primary and secondary minima in the potential energy function.

The results of the stability analysis are best presented as a stability plane $\kappa-N_{\mathrm{G}}$, with the degree of retardation, the repulsion number, and the particle size ratio as parameters. In all cases the stability plane is divided into three regions, as shown in Fig. 3. The spheres can either be flocculated in the primary or secondary minimum or remain dispersed. In order to properly interpret the physical process Fig. 3 represents, consider a situation in which doublets are initially present in a suspension at normal gravity $\left(N_{\mathrm{G}} \simeq 50\right)$ for $\kappa=400$. After flocculation in the secondary minimum the particle pair aligns itself with the $\theta=\pi$ axis. Then, if the suspension is centrifuged at a gradually increasing rate $\left(N_{\mathrm{G}} \propto g\right)$, the doublets will first deflocculate out of the secondary minimum and then flocculate into the primary minimum at higher centrifugal rates, while remaining stable against gravity-induced flocculation at moderate centrifugal rates. It should be noted that while one can experimentally precede from left to right in the stability plane, the reverse procedure (i.e., lowering the centrifugal rate with an initial primary doublet) will not cause deflocculation.

Figure 4 shows that the stability criteria are quite sensitive to changes in the surface po-

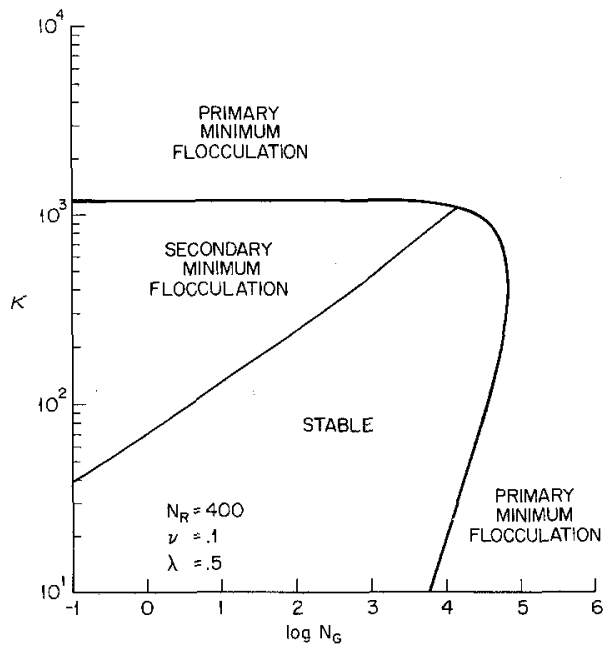

FIG. 3. Gravity-induced flocculation stability plane. 


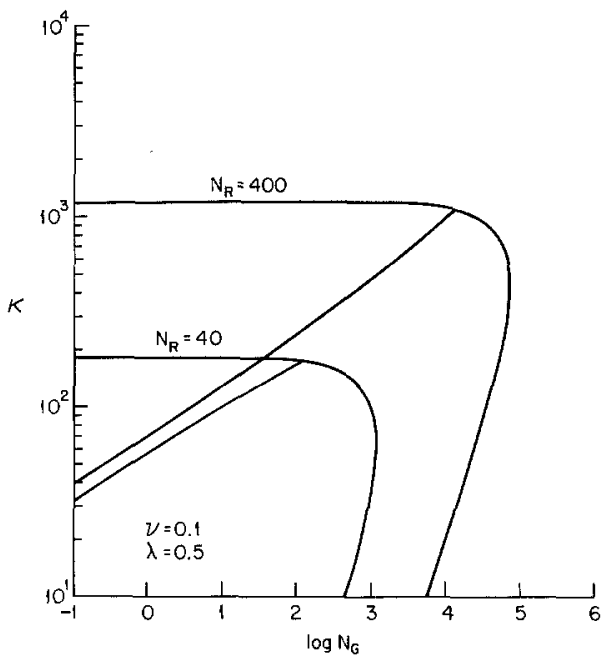

FIG. 4. Effect of surface potential on the gravity-induced flocculation stability plane.

tential $\Psi_{0}\left(\propto N_{\mathrm{R}}^{1 / 2}\right)$. Increasing $\Psi_{0}$ by a factor of approximately 3.2 increases the minimum value of $N_{\mathrm{G}}$ required for primary minimum flocculation by a factor of approximately 32 . Increasing $\Psi_{0}$ increases the repulsive force, thereby resulting in higher critical values of $\kappa$ and $N_{G}$ necessary for primary minimum flocculation to occur. A similar effect is found on secondary minimum flocculation. For constant $N_{\mathrm{G}}$, the critical $\kappa$ is larger for larger $\Psi_{0}$. This occurs since the depth of the secondary minimum increases with increasing $\Psi_{0}$, thereby requiring more electrolyte to significantly reduce this barrier. For constant $\kappa$, the critical $N_{\mathrm{G}}$ for deflocculation from the secondary minimum decreases with increasing $\Psi_{0}$, indicating that increasing repulsion between particles makes deflocculation easier.

The effect of changes in the retardation parameter $\nu$ on the stability criteria is qualitatively similar to the effect of changes in $\Psi_{0}$. A decrease in $\nu$ decreases the magnitude of $f_{\mathrm{A}}$, thereby resulting in a larger net repulsive force and, therefore requiring a larger gravitational force $N_{G}$ to overcome the potential energy barrier. In addition, a decrease in $\nu$ also increases the amount of electrolyte necessary to reduce the potential barrier so primary minimum flocculation can occur. Both of these effects are a result of the weakening of the long range attractive forces due to retardation.

The effect of different particle size ratios $\lambda$ on the stability criteria is somewhat different from the effect of either $\Psi_{0}$ or $\nu$. While the minimum gravitational force $N_{G}$ necessary for primary minimum flocculation increases with decreasing $\lambda$, due to the increased hydrodynamic resistance to particle approach, the minimum value of $\kappa$ necessary for primary minimum flocculation is independent of $\lambda$.

The dominant role interparticle forces play in the stability criteria can be seen by comparing Figs. 2 and 3. The shape of the curve dividing the primary flocculation region from the other two in Fig. 3 is nearly identical to the shape of the curves shown in Fig. 2. In fact, because interparticle forces are so important in flocculation processes, stability results similar to those displayed in Fig. 3 are also found in shear-induced flocculation (5, 7). That is, lyophobic colloids can be unstable at low and high shear rates, but stable against shear-induced flocculation at intermediate rates. There appears to be, however, one significant difference. In gravity-induced flocculation particle capture can occur at low and high electrolyte concentrations for certain gravitational forces (e.g., in Fig. 3 for $10^{3.7}$ $<N_{\mathrm{G}}<10^{4.8}$ ), but not at intermediate $\kappa$ values. In shear-induced flocculation this phenomena does not seem to take place, as can be seen from Fig. 7 in (5). However, since van de Ven and Mason (5) did not carry out calculations for low electrolyte concentrations (i.e., for $\kappa$ $<\sim 100.0$ ), one cannot be totally sure.

\section{Kinetics of Gravity-Induced Flocculation}

In this section the effects of the five dimensionless parameters $\left(N_{\mathrm{G}}, \lambda, \kappa, N_{\mathrm{R}}, \nu\right)$ on the gravity-induced capture efficiency $\alpha_{\mathrm{Gr}}$ are discussed. The range of values taken for these parameters apply to aqueous suspensions with particles on the order of $2 \mu \mathrm{m}$.

Negligible electrostatic repulsion. The effect of gravitational forces $N_{\mathrm{G}}$ on $\alpha_{\mathrm{Gr}}$, in the absence of electric double layer repulsion, is 


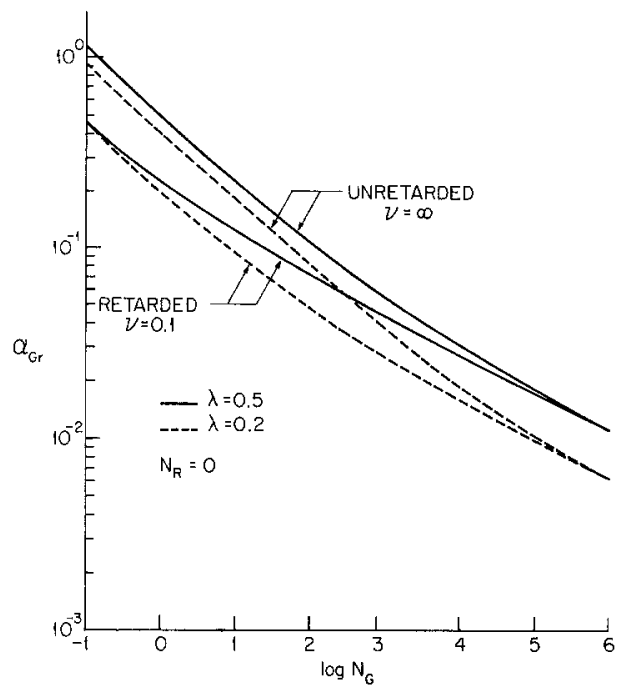

FIG. 5. Effect of gravity on the gravity-induced capture efficiency for various degrees of retardation and particle size ratios in the absence of electric double layer repulsion.

shown in Fig. 5. It is evident from this figure that hydrodynamic interactions and a longrange attractive force affect the rapid flocculation rate in opposing ways. For weak gravitational forces, $N_{\mathrm{G}}<0.1$, the attractive force can actually enhance the collision rate $(\lambda=0.5$ and $\nu=\infty$ ) so that $\alpha_{\mathrm{Gr}}>1.0$. For the most part, however, the strong lubrication stresses generated at small interparticle separations diminish the flocculation rate and $\alpha_{\mathrm{Gr}}<1.0$. For large gravitational forces $\alpha_{\mathrm{Gr}}$ is fairly independent of the retardation parameter $\nu$, which allows one to use the following empirical expression for many practical purposes:

$$
\alpha_{\mathrm{Gr}}=f(\lambda) N_{\mathrm{G}}^{-0.20},
$$

where $f(\lambda)$ equals 0.10 for $\lambda=0.2$ and 0.17 for $\lambda=0.5$. Combining [1]-[3], [21], and [25], one finds that $\phi_{\mathrm{Gr}}$ is approximately proportional to $g^{0.80}$ and not $g$ as predicted by Saffman and Turner (11). This 0.80 power dependence has also been found in shear-induced flocculation $(6,7)$. However, considering the physical differences between the two flocculation processes, this numerical similarity should be considered no more than coincidence.
Varying the particle size ratio, while holding the gravitational force constant, in the absence of electrostatic repulsion leads to the results shown in Fig. 6. As expected, for small values of $N_{\mathrm{G}}$ the gravity-induced capture efficiency is fairly independent of $\lambda$, as shown in Fig. 5 for $\nu=0.1$. However, for larger values of $N_{\mathrm{G}}$ a maximum capture rate occurs at $\lambda \simeq 0.42$. When $\lambda$ is very small, the settling of the larger sphere is unaffected by the presence of the smaller sphere. The smaller sphere then follows the streamlines of flow around the larger particle unless the interparticle distance is small enough at some point for particle capture to occur as a result of attractive forces. Consequently, for very small $\lambda$, the capture efficiency is satisfactorily given by (33):

$$
\alpha_{\mathrm{Gr}}(\lambda \rightarrow 0) \simeq \frac{2.13 \lambda^{4 / 3}}{N_{\mathrm{G}}^{1 / 3}}
$$

which indicates that $\alpha_{\mathrm{Gr}}$ approaches zero as $\lambda$ approaches zero. When $\lambda=1$, no particle capture can occur due to differential creaming since the particles are settling at the same rate. However, in this case the London van der Waal forces can still bring the particles together, thereby resulting in a finite value of $\alpha_{\mathrm{Gr}}$ for $\lambda=1$. As $\lambda \rightarrow 1$, however, the trajectory model becomes unrealistic since the effect of

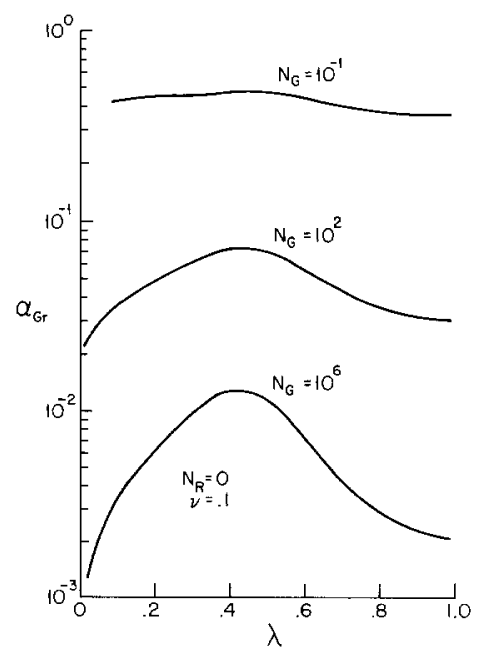

FiG. 6. Effect of increasing particle size ratio on the gravity-induced capture efficiency for various gravitational forces in the absence of electric double layer repulsion. 
Brownian diffusion or particle inertia would then dominate the interparticle forces at large distances. Consequently, particle capture for $\lambda=1$ may not occur.

Particle size ratio effects in shear-induced flocculation are completely different from those found in gravity-induced flocculation. In shear flow, Adler (9) predicts a monotonically increasing capture rate with increasing $\lambda$, in contrast to the presence of a maximum capture rate found in the present work.

Significant electrostatic repulsion. When electric double layer repulsion is included in the analysis, some interesting results are obtained. The first of these are shown in Fig. 7, where slight changes in the electrolyte concentration give rise to significantly different types of behavior as the gravitational force is increased. For large values of $\kappa$ the capture efficiency is not affected since the double layer is essentially collapsed. For lower electrolyte concentrations the capture efficiency still remains unaffected for low values of $N_{\mathrm{G}}$; for example, when $\kappa=400, \alpha_{\mathrm{Gr}}(\kappa=400)=\alpha_{\mathrm{Gr}}$ $(\kappa=2000)$ for $N_{\mathrm{G}}<\simeq 10^{2.8}$. However, as $N_{\mathrm{G}}$ is increased for these lower values of $\kappa$, the conditions necessary for particle capture become very unfavorable and, consequently, $\alpha_{\mathrm{Gr}}$ $=0$ above some critical gravitational force

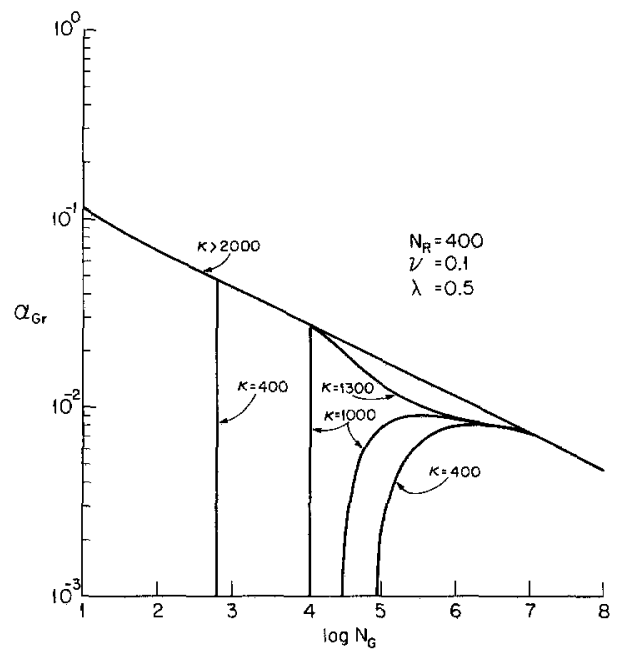

FiG. 7. Effect of gravity on the gravity-induced capture efficiency for various electrolyte concentrations.
$\left(N_{\mathrm{G}}\right)_{\mathrm{cl}}$. Further increasing the gravitational force has no effect on the capture efficiency until a second critical gravitational force is reached above which $\alpha_{\mathrm{Gr}}$ increases until a maximum value is reached, followed by a decrease. This effect is in agreement with the stability analysis presented previously, which shows that a colloidal suspension can flocculate at low and high gravitational forces, but remain stable against sedimentation flocculation at intermediate gravitational forces. For magnitudes of gravity less than $\left(N_{\mathrm{G}}\right)_{\mathrm{c}_{1}}$ flocculation occurs in the secondary minimum, and in the primary minimum for gravitational forces larger than $\left(N_{\mathrm{G}}\right)_{\mathrm{c}_{2}}$.

This effect of different electrolyte concentrations on the particle capture efficiency is also found in shear-induced flocculation (6), which is another indication of the importance interparticle forces play in flocculation processes. Particle convection, whether its by differential creaming or shear flow, is primarily important in bringing the particles close enough to induce flocculation if the interparticle interactions are favorable.

The influence of hydrodynamic interactions on the flocculation process can be illustrated by looking at their effect on the magnitude of the two critical gravitational forces $\left(N_{\mathrm{G}}\right)_{\mathrm{c}_{i}}(i$ $=1,2$ ) just discussed (see Fig. 8). As $\lambda$ is increased the hydrodynamic resistance to relative particle motion decreases, thereby requiring smaller gravitational forces to cause deflocculation and to induce primary minimum flocculation.

Another interesting effect of double layer repulsion on the capture efficiency is shown in Fig. 9, where the electrolyte concentration is varied while the gravitational force is kept constant. For small gravitational forces $\left(N_{\mathrm{G}}\right.$ $=10^{2}$ and $\left.10^{3}\right)$ there is no particle capture $\left(\alpha_{\mathrm{Gr}}=0\right)$ until a critical electrolyte concentration $\kappa_{\mathrm{c}}$ is reached, above which $\alpha_{\mathrm{Gr}}$ remains constant. For intermediate values of $N_{\mathrm{G}}$, flocculation can occur at low and high electrolyte concentrations, but not under moderate concentrations. Whereas, for large gravitational forces, flocculation occurs at all electrolyte 


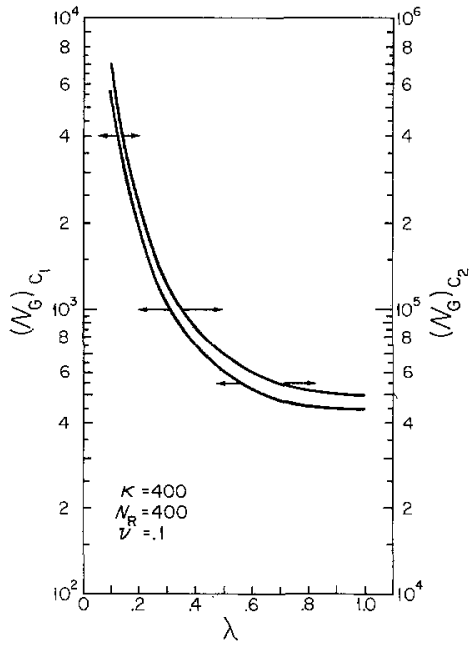

FIG. 8. Effect of increasing particle size ratio (or decreasing hydrodynamic resistance) on the critical gravitational forces. The critical force $\left(N_{\mathrm{G}}\right)_{\mathrm{c} 1}$ is the gravitational force necessary to deflocculate a particle pair from the secondary minimum, while $\left(N_{\mathrm{G}}\right)_{\mathrm{c}_{2}}$ is the gravitational force necessary to overcome the potential energy barrier so primary minimum flocculation can occur.

concentrations, with a minimum rate occuring at some particular value of $\kappa$.

The initial decrease in the capture efficiency for both intermediate and large values of $N_{\mathrm{G}}$ is the result of an increasing repulsive barrier with increasing $\kappa$ (see Fig. 2). In fact, as $\kappa$ is increased above some critical $\kappa_{\mathrm{c}}$ for intermediate values of $N_{G}$, the gravitational force will not be large enough to overcome the potential energy barrier. Consequently, $\alpha_{\mathrm{Gr}}=0$ in this region (i.e., the redispersion region shown in Fig. 3) until $\kappa$ is sufficiently large for the double layer to be nearly collapsed. For the larger gravitational forces the capture efficiency continually decreases with increasing $\kappa$ until a certain value of $\kappa$ at which particle/ particle encounters occur at a close enough interparticle distance (due to the compression of the double layer) so that attractive forces start to dominate the repulsive forces, thereby resulting in an increase in $\alpha_{\mathrm{Gr}}$ with increasing $\kappa$.

The effect of increasing the electrolyte concentration for a fixed gravitational force has both similarities and differences with the case of shear-induced flocculation (6). For small flow parameters, both cases exhibit a critical electrolyte concentration, below which there is no particle capture and above which the capture rate is constant. However, it is with the larger flow parameters that the differences between gravity- and shear-induced flocculation become apparent. In shear-induced flocculation there appears to be no prediction of particle capture for low electrolyte concentrations (6). Therefore, the phenomena of flocculation at low and high electrolyte concentrations for intermediate $N_{\mathrm{G}}$, and the presence of a minimum flocculation rate for large $N_{\mathrm{G}}$, appears to be unique to the process of gravity-induced flocculation. However, as pointed out in the stability criteria section, van de Ven and Mason (6) did not carry out calculations for low electrolyte concentrations. Consequently, one cannot be totally sure of this difference.

It should be noted that the effect of the surface potential $\Psi_{0}$ (through the dimensionless parameter $N_{R}$ ) on the capture efficiency is similar to $\kappa$, but occurs in the reverse direction. That is, decreasing $\Psi_{0}$ is analogous to increasing $\kappa$.

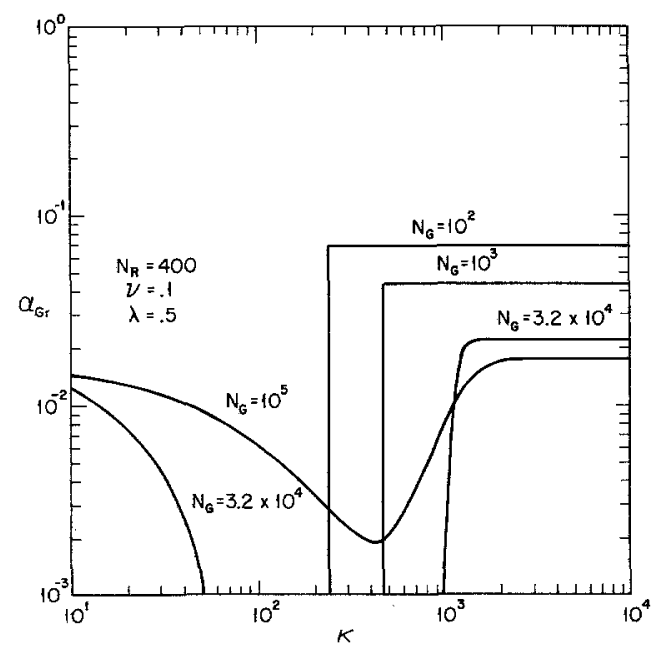

FIG. 9. Effect of increasing electrolyte concentration on the gravity-induced capture efficiency for various gravitational forces.

Journal of Colloid and Interface Science, Vol, 101, No. 1, September 1984 


\section{SUMMARY}

In order to obtain a realistic model of gravity-induced flocculation, we have rigorously accounted for the effects of gravitational and interparticle (both dispersion and electrostatic) forces, as well as hydrodynamic interactions. This model enables one to compute the gravity-induced flocculation collision kernel for any given particle sizes, chemical and physical condition. These collision kernels can be incorporated into the general population balance equations for systems undergoing both creaming and gravity-induced flocculation (34, 35 ). The solution of this coupled system of balance equations would allow one to accurately predict changes in both the particle size distribution and the total particle concentration as a function of time and position in a reaction system (provided the particles are large enough to neglect Brownian flocculation). Being able to accurately predict changes in colloidal systems allows one to determine the experimental conditions necessary for the desired degree of stability. For example, manufacturers of polymer latexes want to maintain stability in order to avoid significant flocculation, which could lead to poor quality products. On the other hand, pharmaceutical manufacturers want to increase the flocculation rate, thereby allowing them to separate finely divided solids from the suspending medium.

\section{NOTATION}

$\begin{array}{ll}A & \text { Hamaker constant } \\ a_{i} & \text { Particle radius } \\ D_{0} & \text { Stokes-Einstein relative diffusion co- } \\ & \text { efficient }=\left(k T / 6 \pi \mu_{\mathrm{f}}\right)\left(1 / a_{1}+1 / a_{2}\right) \\ F_{\text {int }} & \text { Total dimensionless interparticle force } \\ g & \text { Local acceleration of gravity } \\ K & \text { Reciprocal of the double layer thick- } \\ & \quad \text { ness } \\ k & \text { Boltzmann's constant } \\ N_{0 i} & \text { Concentration of particles of size } a_{i} \\ R & \quad \text { Dimensionless interparticle distance } \\ & \quad=2 r /\left(a_{1}+a_{2}\right) \\ r & \text { Distance between particle centers }\end{array}$

$T$

$t$

$u_{0 i} \quad$ Stokes creaming rate for particles of size $a_{i}=2 g \Delta \rho a_{i}^{2} / 9 \mu_{\mathrm{f}}$

$V_{\mathrm{A}} \quad$ London van der Waal attractive potential

$V_{\mathbf{R}} \quad$ Electric double layer repulsive potential

$V_{\text {int }}$ Total interparticle potential $=V_{\mathrm{A}}$ $+V_{R}$

v Relative sphere velocity

$\mathbf{v}_{\mathrm{Br}} \quad$ Relative sphere velocity due to Brownian motion

Relative sphere velocity due to gravity

$\begin{array}{ll}\mathbf{v}_{\mathrm{g}} & \text { Relative sphere velocity due to inter- } \\ \mathbf{v}_{\mathrm{int}} & -\end{array}$ particle forces

$W_{\mathrm{Gr}} \quad$ Gravity-induced flocculation stability ratio

$X_{i} \quad$ Cartesian coordinate

$\mathcal{A}(R)$ Hydrodynamic correction to Stokes relative creaming rate $\left(u_{02}-u_{01}\right)$ for particles moving parallel to their line-of-centers

$\mathscr{B}(R) \quad$ Hydrodynamic correction to Stokes relative creaming rate $\left(u_{02}-u_{01}\right)$ for particles moving perpendicular to their line-of-centers

$G(R)$ Hydrodynamic correction to StokesEinstein relative diffusion coefficient $D_{0}$ for particles moving parallel to their line-of-centers

$\phi_{\mathrm{Gr}}^{0} \quad$ Gravity-induced single particle flocculation rate when interparticle forces and hydrodynamic interactions are neglected

$\partial_{\mathrm{Gr}} \quad$ Gravity-induced single particle flocculation rate when interparticle forces and hydrodynamic interactions are included

$\begin{array}{cl}\ell & \text { Radius of capture cross-section area } \\ f_{\mathrm{A}} & \text { Dimensionless London van der Waal }\end{array}$ attractive force

$f_{\mathrm{R}} \quad$ Dimensionless electric double layer repulsive force

$N_{\text {A }} \quad$ Dimensionless parameter describing the relative importance of London van der Waal attractive forces to system thermal forces 
$N_{\mathrm{G}} \quad$ Dimensionless parameter describing the relative importance of gravitational forces to London van der Waal attractive forces

$N_{\mathrm{R}} \quad$ Dimensionless parameter describing the relative importance of electrostatic repulsive forces to London van der Waal attractive forces

$\alpha_{\mathrm{Gr}} \quad$ Gravity-induced flocculation capture efficiency

$\theta \quad$ Azimuthal coordinate-spherical coordinate system

$\kappa \quad$ Dimensionless reciprocal of the electric double layer thickness $=K\left(a_{1}\right.$ $\left.+a_{2}\right) / 2$

$\lambda \quad$ Particle size ratio $=a_{1} / a_{2}(0<\lambda$ $\leqslant 1)$

$\lambda_{\mathrm{L}} \quad$ London wavelength representing the characteristic wavelength of the dispersion interaction

$\mu_{\mathrm{f}} \quad$ Viscosity of the suspending medium

$\nu \quad$ Dimensionless electromagnetic retardation parameter

$\Delta \rho \quad$ Density difference between the particles and the suspending medium

$\phi \quad$ Polar coordinate - spherical coordinate system

$\Psi_{0} \quad$ Surface potential

\section{REFERENCES}

1. Derjaguin, B. V., and Muller, V. M., Dokl. Akad. Nauk. S.S.S.R. (English Translation), 176, 738 (1967).

2. Spiclman, L. A., J. Colloid Interface Sci. 33, 562 (1970).

3. Curtis, A. S. G., and Hocking, L. M., Trans. Faraday Soc. 66, 1381 (1970).

4. Roebersen, G. J., and Wiersema, P. H., J. Colloid Interface Sci. 49, 98 (1974).

5. van de Ven, T. G. M., and Mason, S. G., J. Colloid Interface Sci. 57, 505 (1976).

6. van de Ven, T. G. M., and Mason, S. G., Colloid Polym. Sci. 255, 468 (1977).

7. Zeichner, G. R., and Schowalter, W. R., AIChE J. 23, 243 (1977).

8. Zeichner, G. R., and Schowalter, W. R., J. Colloid Interface Sci. 71, 237 (1979).
9. Adler, P. M., J. Colloid Interface Sci. 83, 106 (1981).

10. Feke, D. L., and Prabhu, N., Presented at the AIChE 1982 Annual Meeting, Los Angeles, Ca. (Nov. 14-19).

11. Saffman, P. F., and Turner, J. S., J. Fluid Mech. 1, 16 (1956).

12. Wacholder, E., and Sather, N. F., J. Fluid Mech. 65, 417 (1974).

13. Hocking, L. M., and Jonas, P. R., Q. J. Roy. Met. Soc. 96, 722 (1970).

14. Klett, J. D., and Davis, M. H., J. Atmos. Sci. 30, 107 (1973).

15. Beard, K. V., and Grover, S. N., J. Atmos. Sci. 31, 543 (1974).

16. Beard, K. V., J. Atmos. Sci. 31, 1595 (1974).

17. Wang, P. K., Grover, S. N., and Pruppacher, H. R., J. Atmos. Sci. 35, 1735 (1978).

18. Rajagopalan, R., Ph.D. Thesis, Syracuse University (1974).

19. Rajagopalan, R., and Tien, C., Canad. J. Chem. Eng. 55, 246 (1977).

20. Happel, J., and Brenner, H., "Low Reynolds Number Hydrodynamics." Noordhoff International Publishing, Leyden, The Netherlands, 1965.

21. Melik, D. H., and Fogler, H. S., J. Colloid Interface Sci. 101, 84 (1984).

22. Derjaguin, B., and Landau, L., Acta. Physiochim. 14, 633 (1941).

23. Verweey, E. J. W., and Overbeek, J. Th. G., "Theory of the Stability of Lyophobic Colloids." Elsevier, Amsterdam, 1948.

24. Hamaker, H. C., Physica 4, 1058 (1937).

25. Ho, N. F. H., and Higuchi, W. I., J. Pharm. Sci. 57, 436 (1968).

26. Gregory, J., Disc. Faraday. Soc. 42, 168 (1966).

27. Hogg, R., Healy, T. W., and Fuerstenau, D. W., Trans. Faraday Soc. 62, 1638 (1966).

28. Barouch, E., Matijevic, E., Ring, T. A., and Finlan, J. M., J. Colloid Interface Sci. 67, 1 (1978).

29. Ohshima, H., Chan, D. Y. C., Healy, T. W., and White, L. R., J. Colloid Interface Sci. 92, 232 (1983).

30. Davis, M. H., Chem. Eng. Sci. 24, 1769 (1969).

31. O'Neill, M. E., and Majumdar, S. R., Z. Angew. Math. 21, 164 (1970).

32. Melik, D. H., Ph.D. Thesis, University of Michigan, (1984).

33. Davis, R. H., J. Fluid Mech., 1984 (in press).

34. Reddy, S. R., Melik, D. H., and Fogler, H. S., J. Colloid Interface Sci. 82, 116 (1981).

35. Reddy, S. R., and Fogler, H. S., J. Colloid Interface Sci. 82, 128 (1981). 